\title{
A mesoscopic Archimedean tiling having a new complexity in an $A B C$ star polymer
}

\author{
AUTHOR(S): \\ Takano, A; Kawashima, W; Noro, A; Isono, Y; \\ Tanaka, N; Dotera, T; Matsushita, Y
}

\section{CITATION:}

Takano, A ... [et al]. A mesoscopic Archimedean tiling having a new complexity in an ABC star polymer. Journal of Polymer Science Part B: Polymer Physics 2005, 43(18): 2427-2432

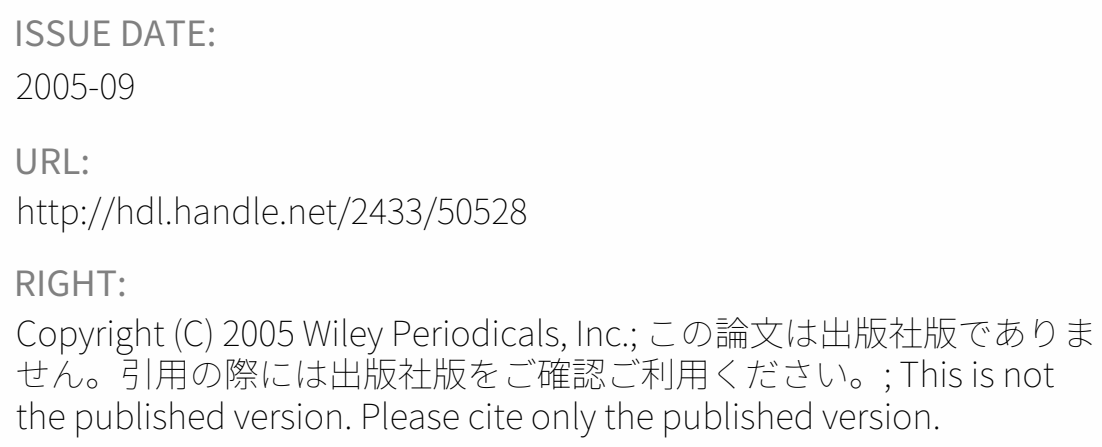




\section{POLYMER PHYSICS PRIZE}

awarded to:

Atsushi Takano, Wataru Kawashima, Atsushi Noro, Yoshinobu Isono, Nobuo

Tanaka, Tomonari Dotera, Yushu Matsushita for their paper:

"A Mesoscopic Archimedean Tiling Having a New Complexity in an ABC Star Polymer"

Volume 43, Issue 18, Pages 2427-2432 (2005) Journal of Polymer Science Part B: Polymer Physics

\section{A Mesoscopic Archimedean Tiling} Having a New Complexity in an ABC Star Polymer

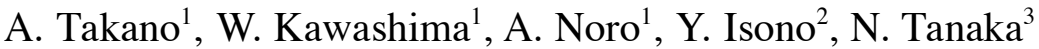

T. Dotera ${ }^{4} \&$ Y. Matsushita ${ }^{1 *}$

${ }^{1}$ Department of Applied Chemistry, Graduate School of Engineering, Nagoya

University, Furo-cho, Chikusa-ku, Nagoya 464-8603 Japan

${ }^{2}$ Department of Chemistry, Nagaoka University of Technology, Kamitomioka-cho,

Nagaoka, Niigata 940-2188 Japan

${ }^{3}$ Ecotopia Science Institute, Nagoya University, Furo-cho, Chikusa-ku, Nagoya 464-8603 Japan

${ }^{4}$ Department of Polymer Chemistry, Graduate School of Engineering, Kyoto University, Katsura, Nishikyo-ku, Kyoto 615-8510 Japan

*To whom correspondence and requests for materials should be addressed.

E-mail: yushu@apchem.nagoya-u.ac.jp

\section{Key Words}

Archimedean tiling, $\mathrm{ABC}$ star-branched terpolymer, mesoscale self-organization, hierarchy, $\left(3^{2} \cdot 4 \cdot 3.4\right)$ 


\begin{abstract}
The Archimedean tiling $\left(3^{2} .4 .3 .4\right)$ is a regular but complex polygonal assembly of equilateral triangles and squares. This tiling pattern with mesoscopic repeating distance has been found for an $\mathrm{ABC}$ star-branched three-component polymer composed of polyisoprene, polystyrene and poly (2-vinylpyridine). In this structure the environment of a molecule splits into multiple sites and two microdomains with different sizes and shapes are formed for one component. This complexity is the first observation in complex polymer systems and can lead to a new type of mesoscale self-organization. The tiling pattern has been observed for the other materials on much shorter length-scale, therefore, the experimental fact observed in the present study is demonstrating that the complexity is universal over different hierarchy.
\end{abstract}


Soft materials consisting of different chemical/physical species in a molecule such as lipids ${ }^{1}$, amphiphiles ${ }^{2}$ block copolymers ${ }^{3}$ and copolymer-based supramolecules ${ }^{4}$ are well known to form periodic structures spontaneously because of their intramolecular segregation and self-assembling nature. In addition to classical structures like BCC-packed spheres, hexagonally-packed cylinders and lamellar structures found in early days, ${ }^{5}$ extraordinary periodic structures, i.e., bicontinuous- ${ }^{6}$ and tricontinuous double Gyroid, ${ }^{7}$ plumber's nightmare ${ }^{8}$ etc. have been found one after another for various soft material systems. Summarizing all the results, we notice that the environment of an element, a lipid or a polymer, is almost the same in these systems.

$\left(3^{2} .4 .3 .4\right)$ is a complex but regular polygonal assembly of equilateral triangles and squares, which is one of the eleven Archimedean tiling patterns (Figure 1A and the caption). ${ }^{9}$ It has been known that the tiling appears in a layered crystalline structure for rather complex metallic alloys ${ }^{10}$ known as Frank-Kasper phases. ${ }^{11}$ Recently a $\left(3^{2} .4 .3 .4\right)$ structure scaled up more has been found in a chalcogenide, ${ }^{12}$ followed by further scaled-up supramolecular micellar complex phases of organic dendrons. ${ }^{13}$ These complex phases are "complex" in the following meaning: the environment of an element, an atom or a micelles, in a complex phase splits into several sites, that is, the coordination numbers are different, ${ }^{11,13}$ even though the elements are the same. We intend to step up one order more by exploring into the polymeric system, where the characteristic length reaches to the wavelength of visible light region.

In order to construct such a tiling with mesoscopic scale, we have studied a multi-component polymer system. In ABC star-shaped block terpolymer systems, true two-dimensional tilings can be self-organized because junction points should be aligned one-dimensionally under geometrical restriction (Figure 1B), being assumed that three polymer chains are totally incompatible and all chains are long enough. For this three-component polymeric system, theoretical work by Dotera et al. pointed out the formation of hexagonal $\left(6^{3}\right)$ pattern, ${ }^{14}$ while Bohbot-Raviv and Wang reported on $\left(6^{3}\right)$ and $\left(4.8^{2}\right)$ structures. ${ }^{15}$ Later it has been summarized that only three patterns, $\left(6^{3}\right),\left(4.8^{2}\right)$ and (4.6.12), are allowed to exist, since only three polygons should meet on a vertex and only even polygons should appear proposed as the even polygon theorem. ${ }^{16}$ OC瞘VSTF 賑

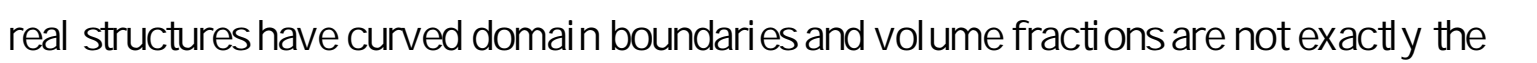

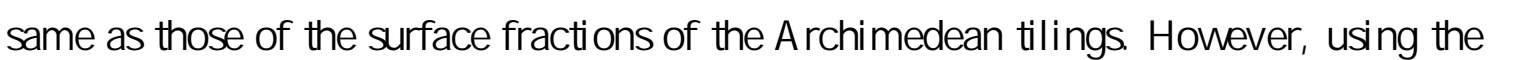




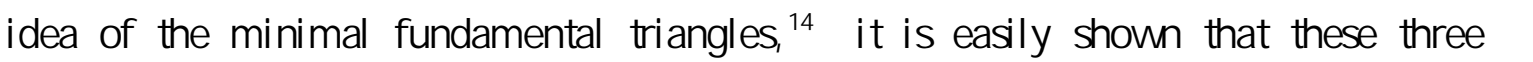

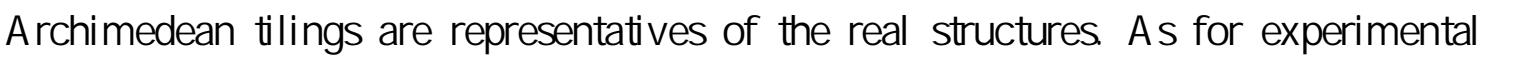

STVNI, $\left(4.8^{2}\right)$ structure was observed from two different systems ${ }^{17,18}$ and a family of (4.6.12) which looks like (3.4.6.4) structure was also reported for the other ABC-star molecule, ${ }^{19}$ while co-axial structures were reported for another system. ${ }^{20}$ Recently, three patterns, $\left(6^{3}\right),\left(4.8^{2}\right)$ and (4.6.12) have been observed systematically at a time with changing compositions of $\mathrm{ABC}$ molecules. ${ }^{21}$ Thus, if we regard polygons as polymeric domains directly tiled, a complex Archimedean tilings ( $3^{2}$.4.3.4) cannot exist. However, if we employ a tiling as a skeleton, the argument is different. In this paper, we show experimental evidence of a mesoscopic $\left(3^{2} .4 .3 .4\right)$ phase together with the corresponding simulated results.

Parameter $x$ is introduced to represent the important molecular volumetric character. Namely $x$ is the volume fraction of component $\mathrm{P}$ when volume ratios of three components, I:S:P, are expressed by $1: 1: x$, since the volume ratio of component I and $\mathrm{S}$ are designed to be the same. Polymer sample with $x=1.3$ used in this work, $\mathrm{I}_{1.0} \mathrm{~S}_{1.0} \mathrm{P}_{1.3}$, was obtained by blending two I-S-P star-shaped molecules, i.e., $\mathrm{I}_{1.0} \mathrm{~S}_{1.0} \mathrm{P}_{1.2}$ and $\mathrm{I}_{1.0} \mathrm{~S}_{1.0} \mathrm{P}_{1.9}$ at the weight ratio of $0.85 / 0.15$. The details of preparation and characterization methods were described earlier. ${ }^{21}$ It was confirmed in a previous work that the sample with $x=1.2$ shows $\left(4.8^{2}\right)$ two-dimensional pattern, while the sample with $x=1.9$ possesses $(4.6 .12){ }^{21}$ The sample film was given by solvent-casting from dilute solution of tetrahydrofuran followed by drying and annealing at $150^{\circ} \mathrm{C}$ for 4 days in a vacuum oven. The annealing temperature is well above the glass transition temperatures of three component polymers, so that we regard the structure obtained must be a stable one. The annealed sample was stained with osmium tetroxide and cut into ultra thin sections by using an ultra-microtome. TEM observations were carried out by adopting the same manner reported previously. ${ }^{22}$

Figure 2 shows typical TEM images of the sample $\mathrm{I}_{1.0} \mathrm{~S}_{1.0} \mathrm{P}_{1.3}$. It has been confirmed that three polymer chains branched at one junction point all show cylinder-based structure. ${ }^{21}$ Accordingly the image in Figure $2 \mathrm{~A}$ shows the cross section of cylindrical structure, while that in Figure $2 \mathrm{~B}$ is the side view of cylinders together with the cross section. It is obvious from Figure 2A that the darkest I phase is forming two kinds of microdomains whose sizes and shapes are different from each other and both are packed very periodically. The experimental result obtained in the present study is the first 
observation for the chains with the same length splitting into distinctly different microdomains. A complex structure named "the knitting-pattern" was observed by Breiner et al. for a triblock copolymer system, where molecules were distributed into different environment but still within the microdomains with almost the same size and shape. $^{23}$

Figure 3A shows a more enlarged image for this sample. If we observe this figure very carefully, both the darkest I domain and the brightest $\mathrm{S}$ domain are not uniform, while anisotropic gray $\mathrm{P}$ domain is uniform. Here it is evident that the direct tiling patterns such as $\left(4.8^{2}\right)$ and (4.6.12) are not applicable, however, indirect tiling as a skeleton could be given. Then, equilateral triangles and squares were tiled by placing their vertices at the centers of $\mathrm{P}$ domains as shown in Figure 3A. The constructed pattern is indeed the first observed mesoscopic $\left(3^{2} .4 .3 .4\right)$ tiling, where the length of the polygons is about $80 \mathrm{~nm}$. Frank-Kasper phases ${ }^{11}$ and the dendron system ${ }^{13}$ are associated with tetrahedrally closed packing of soft spherical objects such as transition metal atoms or micelles. In contrast to these systems, the present system obviously can be viewed as a pure two-dimensional system and cannot be described by sphere packing. Hence we tentatively explore a more generic scenario to understand why the two-dimensional $\left(3^{2} .4 .3 .4\right)$ could form. Within the framework of a weak crystallization theory, ${ }^{24,25}$ where the cubic term of the density in the Landau expansion is important, we employ a two-component Landau theory proposed by Mermin and Troian. ${ }^{26}$

We can describe density waves for three components as deviations from the averages by using two order parameters, i.e., $\Psi$ and $\Phi$, defined as $\Phi_{\mathrm{I}}=(\Psi+\Phi) / 2$, $\Phi_{\mathrm{S}}=(\Psi-\Phi) / 2, \Phi_{\mathrm{P}}=-\Psi$ under the incompressibility condition: $\Phi_{\mathrm{I}}+\Phi_{\mathrm{S}}+\Phi_{\mathrm{P}}=0$. We consider the third order terms of a Landau free energy of the form $\Psi^{3}$ and $-\Psi^{2} \Phi$. The former term stemming from $-\Phi_{\mathrm{I}} \Phi_{\mathrm{S}} \Phi_{\mathrm{P}}$ represents the effect of junction points such that three components should meet, while the latter term indicates that the largest volume fraction component, $\mathrm{P}$, dominates the transition considering the relationship $\Psi=-\Phi_{\mathrm{P}}$. These terms determine the relation between the sets of reciprocal-lattice vectors forming equilateral or isosceles triangles and the phases of waves in Fourier expansion under the condition of minimizing free energy. Now the form of a generic free energy $f$ describing Archimedean tiling phases to examine is 


$$
\begin{aligned}
& f=\int\left[t \Phi^{2}+\Phi^{4}+\tau \Psi^{2}+\Psi^{4}\right. \\
& \left.+g_{0} \Psi^{3}-g_{1} \Phi \Psi^{2}-g_{2} \Phi^{2} \Psi\right] d \mathbf{r},
\end{aligned}
$$

where $t$ and $\tau$ are parameters corresponding to the temperature, and each $g_{i}$ is a positive parameter. We easily find that reciprocal-lattice vectors with respect to $\left(6^{3}\right)$, $\left(4.8^{2}\right)$ and (4.6.12) can form equilateral or isosceles triangles and naturally contribute to these new third order terms to reduce the free energy. For $\left(3^{2} .4 .3 .4\right)$, phases of waves are completely determined except the two-dimensional translation, and finally $\Psi$ and $\Phi$ are represented as

$$
\begin{aligned}
& \Psi=\cos [2 \pi(2 x)+\pi / 2]+\cos [2 \pi(2 x+y)-\pi / 4] \\
& +\cos [2 \pi(x+2 y)-\pi / 4]+\cos [2 \pi(2 y)+\pi / 2] \\
& +\cos [2 \pi(-x+2 y)+\pi / 4]+\cos [2 \pi(-2 x+y)-\pi / 4]
\end{aligned}
$$

and

$$
\begin{aligned}
& \Phi=\cos [2 \pi(3 x+y)]+\cos [2 \pi(2 x+2 y)+\pi] \\
& +\cos [2 \pi(x+3 y)]+\cos [2 \pi(-x+3 y)-\pi / 2] \\
& +\cos [2 \pi(-2 x+2 y)]+\cos [2 \pi(-3 x+y)+\pi / 2],
\end{aligned}
$$

where the lattice constant is taken to unity and numerical constants are omitted. We then find that these sinusoidal waves can reduce the third order terms, too.

In Figure 3B, the total density wave is displayed together with those of three individual components (Figures 3C-E). The partial area corresponding to the area of Figures C, D and E is surrounded by a white square in lower left of Figure 3B. At lower right of Figures $3 \mathrm{~A}$ and $-3 \mathrm{~B}$, three microdomains are distinguished with characters I, $\mathrm{S}$ and $\mathrm{P}$ to assign their mutual locations. Figure $3 \mathrm{~B}$ is drawn by summing density waves for three components putting the relative contrast factor, i.e., $\Phi_{\mathrm{I}}^{\prime}=a(\Psi+\Phi) / 2$, $\Phi_{\mathrm{S}}{ }^{\prime}=b(\Psi-\Phi) / 2, \Phi_{\mathrm{P}}{ }^{\prime}=-c \Psi$, where 1.0, 0.1 and 0.3 for $a, b$ and $c$ are taken to realize the experimental contrast for microscopy. Two additional adjusting parameters are used to assign all the area into three different phases with three distinct contrasts corresponding to three polymeric phases. Comparing Figure 3B and observed pattern in Figure $3 \mathrm{~A}$, we find that the packing manner of three microdomains in the observed micrograph agrees well with the calculated one.

Furthermore, five molecules are drawn at five locations under different environment in Figure 4A, where every microdomain boundaries are simplified by straight lines instead of curves. Open- and crossed circles correspond to two types of junctions (4.6.10) and (4.8.10), respectively, and we find that their number ratio is 3:2. This figure 
clearly shows that the environment of the present star-shaped terpolymer splits into five distinct sites, consequently it forms two assembling manners, (4.6.10) and (4.8.10). In Figure $4 \mathrm{~b}$ the symmetry elements for five different microdomains, i.e., two I's, two S's and a $\mathrm{P}$ for three polymer phases, are drawn in a unit cell, and the corresponding ones are summarized in Table 1 . From Figure $4 \mathrm{~b}$ and Table 1 , we can easily assign $p 4 g m$ as a two-dimensional space group for this symmetric pattern.

The fact should be stressed that the length of every side of polygons is about $80 \mathrm{~nm}$ and this length is almost one order magnitude longer than that from the supramolecular dendritic molecule, where the characteristic length is ca. $10 \mathrm{~nm}$. The size growth for $\left(3^{2} .4 .3 .4\right)$ tiling shown as metallic alloy $(\sim 0.5 \mathrm{~nm})$, chalcogenide $(\sim 2 \mathrm{~nm})$, supramolecule $(\sim 10 \mathrm{~nm})$ and block terpolymer $(\sim 80 \mathrm{~nm})$ is beautifully demonstrating that the complexity is universal over different hierarchy.

In conclusion mesoscopic $\left(3^{2}\right.$.4.3.4) Archimedean tiling pattern has been observed very clearly as a real-space evidence for a star-branched terpolymer of the $\mathrm{ABC}$ type for the first time.

Acknowledgements: The authors greatly thank to Dr. S. Arai at the Ecotopia Science Institute in Nagoya University for his help in taking transmission electron micrographs. This work was partially supported by the $21^{\text {st }}$ century COE program under support from the Japan Society for the Promotion of Science(JSPS) entitled "The Creation of Nature-Guided Materials Processing" in the School of Engineering, Nagoya University.

References and Notes

1. G. Lindblom, K. Larsson, L. Johansson, K. Fontell, S. Forsen, J. Am. Chem. Soc. 101, 5465 (1979).

2. P.Alexandridis, U. Olsson, B. Lindman, Langmuir 13, 23 (1997).

3. E.L.Thomas, D.M.Anderson, C.S. Henkee, D.Hoffman, Nature 334, 598 (1988).

4. O. Ikkala, G. ten Brinke, Science 295, 2407(2002).

5. M. Matsuo, S. Sagae, H. Asai, Polymer 10, 79(1969).

6. D. A. Hadjuk et al., Macromolecules 27, 4063 (1994)

7. J. Suzuki, M. Seki, Y. Matsushita, J. Chem. Phys. 112, 4862 (2000).

8. A.C. Finnefrock et al., J. Am. Chem. Soc. 125, 13084 (2003). 
9. B.Grunbaum, G. C. Shephard, Tilings and Patterns (Freeman, New York, 1986).

10. G. Bergman, D. P. Shoemaker, Acta Cryst. 7, 857 (1954).

11. F. C. Frank, J. S. Kasper, Acta Cryst. 12, 483 (1959).

12. F. Krumeich, M. Conrad, H.-U. Nissen, B. Harbrecht, Phil Mag. Lett. 78, 357 (1998).

13. X. Zeng, G. Ungar, Y. Liu, V. Percec, A.E. Dulcey, J.K. Hobbs, Nature 428, 157 (2004).

14. T. Dotera, A. Hatano, J. Chem. Phys. 105, 8413(1996).

15. Y. Bohbot-Raviv, Z-G. Wang, Phys. Rev. Lett. 85, 3428(2000).

16. T. Gemma, A. Hatano, T. Dotera, Macromolecules 35, 3225 (2002).

17. H. Huckstadt, A. Gopfert, B. Abetz, Macromol. Chem. Phys. 201, 296 (2000).

18. K. Yamauchi, K. Takahashi, H. Hasegawa, H. Iatrou, N. Hadjichristidis, T. Kaneko, Y. Nishikawa, H. Jinnai, T. Matsui, H. Nishioka, M. Shimizu, H. Furukawa, Macromolecules 36, 6962 (2003).

19. S. Sioula, N. Hadjichristidis, E.L. Thomas, Macromolecules 31, 8429 (1998).

20. S. Sioula, N. Hadjichristidis, E.L. Thomas, Macromolecules 31, 5272 (1998).

21. A. Takano, S. Wada, S. Sato, T. Araki, T. Kazama, S. Kawahara, Y. Isono, A. Ohno,

N. Tanaka, Y. Matsushita Macromolecules 37, 9941(2004)

22. Y. Matsushita, H. Iwata, T. Asari, T.Uchida, G. ten Brinke, A. Takano, J. Chem. Phys. 121, 1129 (2004).

23. U. Breiner, U. Krappe, E.L. Thomas, R. Stadler Macromolecules 31, 135(1998).

24. S. Alexander, J. MacTague, Phys. Rev. Lett. 41, 702 (1974).

25. P. M. Chaikin, T. C. Lubensky, Principles of condensed matter physics (Cambridge University Press, Cambridge, 1995).

26. N. D. Mermin, S. M. Troian, Phys. Rev. Lett. 541524 (1985). 
(a)
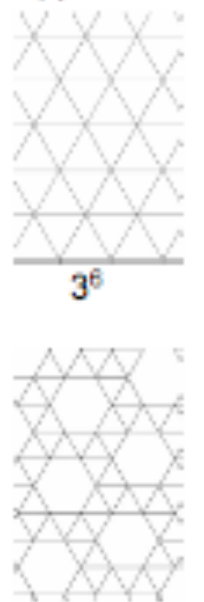

$\left.3^{4} .6\right)$

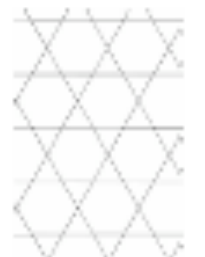

3.6.3.6

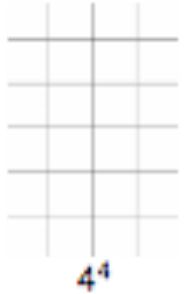

$4^{4}$

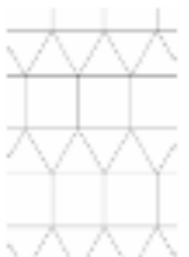

$3^{3} \cdot 4^{2}$

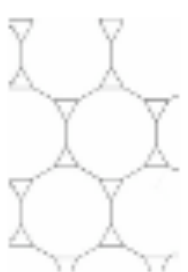

$3.12^{2}$

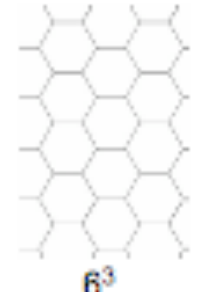

$6^{3}$

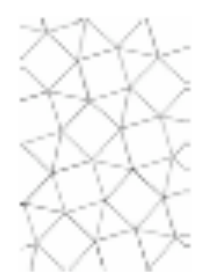

$3^{2} \cdot 4.3 .4$

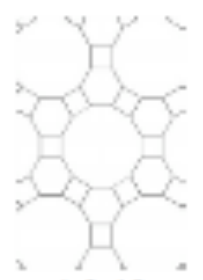

4.6.12 (b)

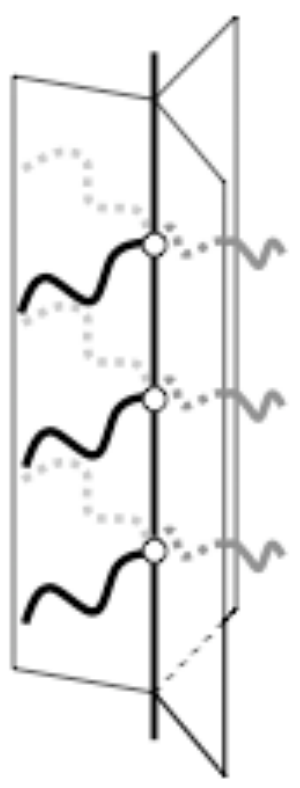

Figure 1 A) Eleven possible Archimedean tilings. The Archimedean tiling is the tessellation of regular polygons provided that all vertices are of the same type. A set of integers $\left(n_{1} . n_{2} . n_{3} \ldots\right)$ denotes a vertex type such that $n_{1}$-gon, $n_{2}$-gon, and $n_{3}$-gon $\ldots$ meet consecutively on each vertex. Superscripts are employed to abbreviate when possible. B) Schematic alignment of ABC star-shaped block terpolymer molecules in a molten state. The junction points have to be aligned on a line and hence no junctions are permitted to stay on the microdomain interfaces, since the strong geometrical restriction being offered to the molecules. 
(a)

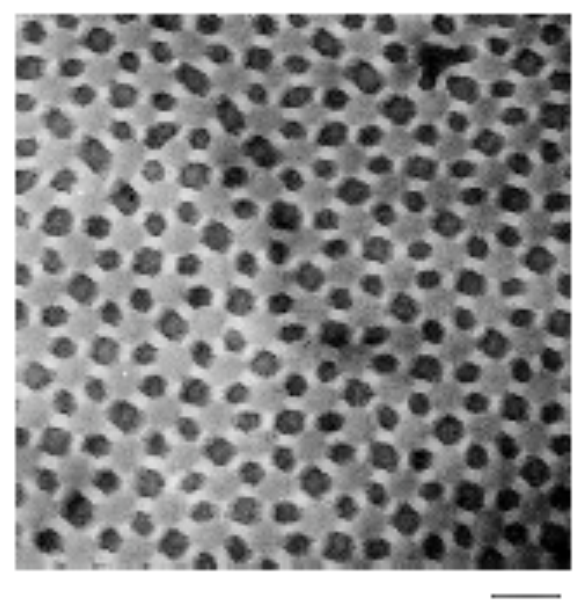

$\overline{100 \mathrm{~nm}}$ (b)

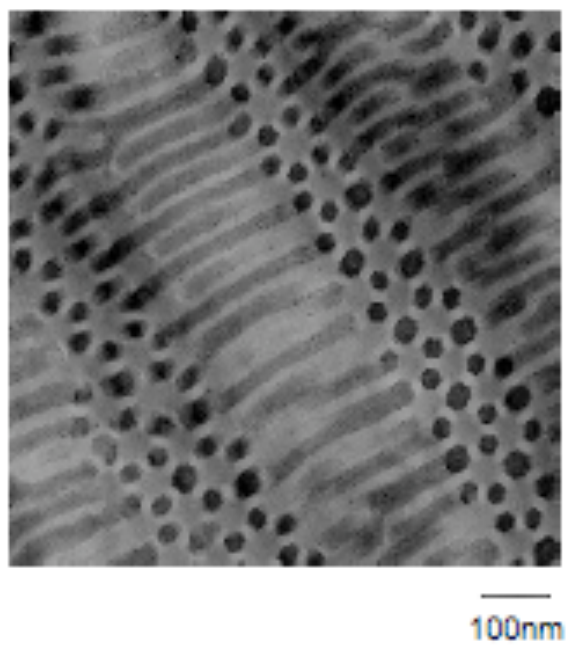

Figure 2 Typical transmission electron micrographs for an I-S-P star-shaped block terpolymer molecule, $\mathrm{I}_{1.0} \mathrm{~S}_{1.0} \mathrm{P}_{1.3}$. Ultra thin sections were cut from edge of the film specimen and the TEM observations were carried out from edge direction. A) The cross section of cylinders packed two-dimensionally and B) the side view of cylinder arrays. 
(a)

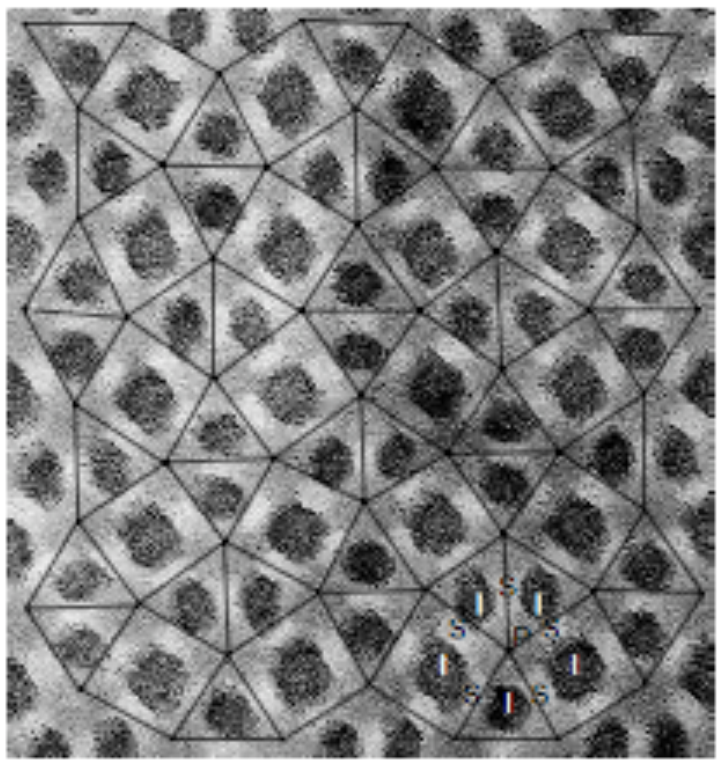

$100 \mathrm{~nm}$

(b)

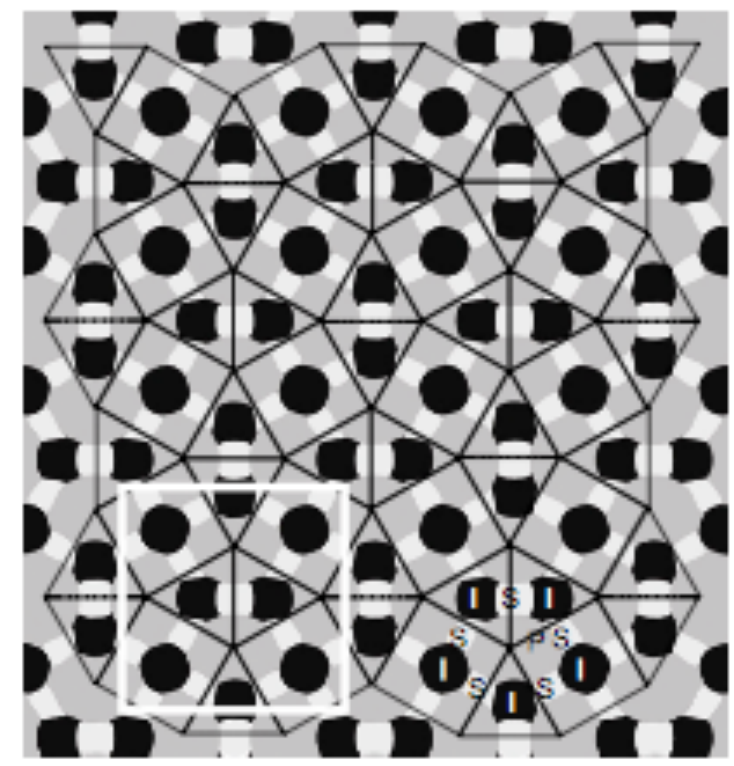

(c)

(d)

(c)
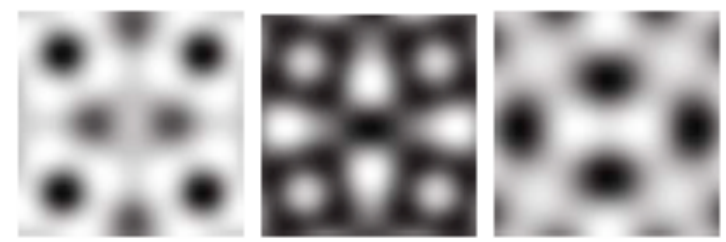

Figure 3 Comparison of domain packing manner in between experiment (A) and calculated pattern (B). One of the Archimedean tiling patterns, ( $3^{2}$.4.3.4), is drawn as thin solid lines throughout in Figures A and B. Figures $\mathrm{C}, \mathrm{D}$ and $\mathrm{E}$ are representing density waves for $\mathrm{I}, \mathrm{S}$ and $\mathrm{P}$ phases, respectively, while Figure B is drawn by summing them. 


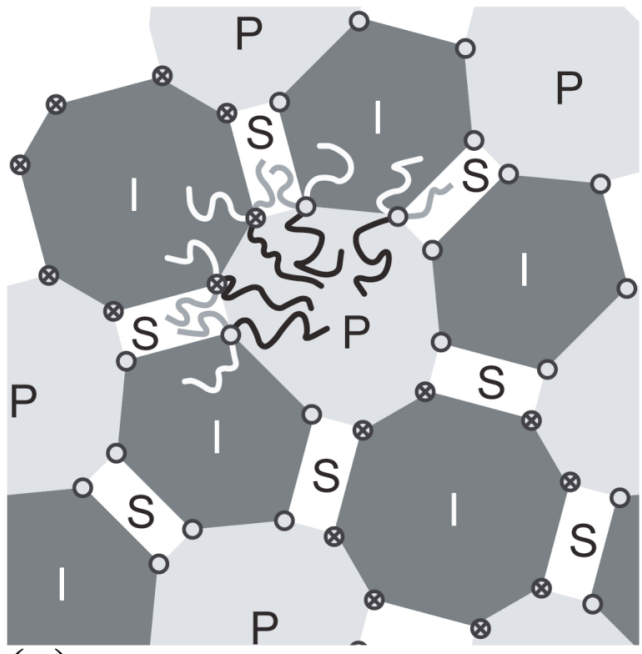

(a)

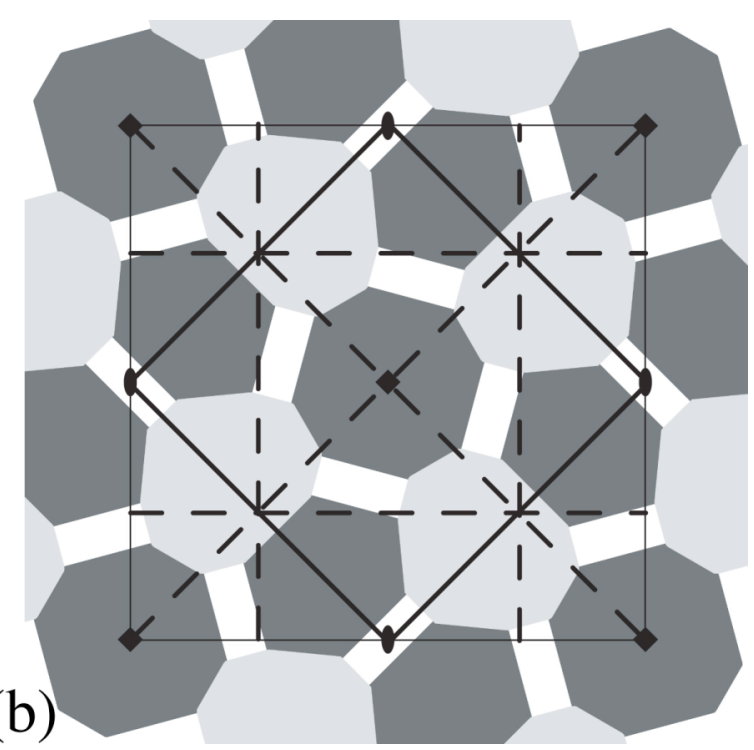

(b)

Figure 4 a) Packing manner of microdomains in detail and five different sites for the molecules. Three microdomains are distinguished with characters I, S and P to assign their mutual locations. The open circles show junction points at the vertices of hexagonal black I domains, while the crossed circles are those of octagonal ones. Five star-shaped molecules are drawn at five different vertices, three of them are sitting on two hexagonal black I domains, whereas another two are on an octagonal one. b) Symmetry elements, i.e., diads, tetrads and symmetry planes, of two-dimensional space group $\mathrm{p} 4 \mathrm{gm}$ in the microphase separated pattern are shown. Solid lines correspond to mirror planes, while dotted lines are axial glide planes.

Table 1 The symmetry elements are summarized.

\begin{tabular}{|l|c|c|}
\hline \multicolumn{1}{|c|}{ Domain type } & $\begin{array}{c}\text { Multiplicity and } \\
\text { Wyckoff letter }\end{array}$ & Site Symmetry \\
\hline (1) Octagonal I domain & $2 \mathrm{a}$ & $4 .$. \\
\hline (2) Hexagonal I domain & $4 \mathrm{c}$ & ..m \\
\hline (3) S domain between two (2)s & $2 \mathrm{~b}$ & $2 \mathrm{~mm}$ \\
\hline (4) S domain between (1) and (2) & $8 \mathrm{~d}$ & 1 \\
\hline (5) P domain & $4 \mathrm{c}$ & ..m \\
\hline
\end{tabular}

